\title{
Classification of Breast Tumors on Digital Mammograms Using Laws' Texture Features
}

\author{
Celia Varela ${ }^{1}$, Nico Karssemeijer ${ }^{2}$, and Pablo G. Tahoces ${ }^{3}$ \\ 1 Department of Radiology, University of Santiago de Compostela, \\ 15782 Santiago de Compostela, Spain \\ mrcuca@usc.es \\ 2 University Hospital Nijmegen, Department of Radiology, \\ PO Box 9101, Nijmegen, 6500 HB, The Netherlands \\ nico@radiology.azn.nl \\ 3 Department of Electronic and Computer Science, \\ University of Santiago de Compostela, \\ 15782 Santiago de Compostela, Spain
}

\section{Introduction}

Mammographic screening is widely used for early detection of breast cancer. Despite the success of screening programs, negative effects should not be underestimated. In many countries, only $15 \%-40 \%$ of detected lesions which are biopsied are subsequently determined malignant. Radiologists might improve their performance, when they could use objective computer-aided diagnosis programs developed with the aim of reducing false positives.

Spiculated margins and irregularly shaped masses are two of the main diagnostic features used by radiologists for identifying potentially malignant lesions. In this study we present a digital image processing algorithm to classify breast lesions based on quantitative measures of tumor shape, contrast, and spiculation.

\section{Material and Methods}

The data set for this study included 131 regions of interest (ROIs). The number of malignant and benign images were 65 and 64 respectively, corresponding to 39 patients for both type of lesions. The ROIs had a pixel size of 100 microns and a depth resolution of 12 bits. Cases were taken from the Galician screening program and from the Digital Database for Screening Mammography (DDSM) [1].

Our study consisted in two main steps:1) Mass segmentation and transformation of its border, 2) Feature extraction and its implementation in a classifier to discriminate between malignant and benign mass.

\subsection{Mass Segmentation and Border Transformation}

The algorithm used for segmentation was an adaptive region growing algorithm[2] . The border region surrounding the mass was transformed into a rectangular band [3]. By this transformation spicules were transformed into vertical lines perpendicular to the mass contour. 
Two low resolution images were obtained by resampling the image by a given factor $(0.7$ and 0.5$)$. In this way, features were extracted at two different resolutions.

\subsection{Feature Extraction and Classification}

Different types of features were extracted from the segmented mass region itself and from its corresponding rectangular band. From the segmented mass region a peak-related and a contrast measure were calculated.

Features based in Laws' texture energy features were extracted from the straighten border region. Three Laws' filters (vertical, horizontal, and symmetrical) were applied to the transformed image. Vertical filter was intended to enhance spiculation, where horizontal filter would suppress them.

From each Laws' image two different features were calculated from the distribution of the gray level values of the pixels in the image: the standard deviation and the skewness. Besides, the ratio of the vertical and horizontal features was also calculated. The filters were applied to the original transformed border region and also to its low-resolution versions. Thus, 24 features were extracted from the rectangular region. Therefore, a total of 26 features were extracted for each ROI.

The classifier used in this study was a three layered feed-forward network using backpropagation as learning algorithm. A leave-one-case-out method was used to train and test the generalization capability of the classifier. The classifier performance was evaluated with receiver operating characteristic (ROC) analysis.

\section{Results}

The best results corresponded to an area under the ROC curve of 0.87 . The classifier used as input features extracted from the highest resolution image joined with the peak-related and the contrast feature. Future work is planned to develop new features and also to enlarge the database.

\section{References}

1. Heath, M., Bowyer, K.W., Kopans, D., et al.: Current status of the Digital Database for Screening Mammography. In: Karsseimeijer, N., Thijssen, M., Hendriks, J.,van Erning, L. (eds). Digital Mammography, Kluwer Academic Publishers (1998) 457460.

2. Tahoces, P.G., Varela, C., Méndez, A.J., Souto, M., Vidal, J.J.: An automatic algorithm for segmentation of mammographic masses on a computerized detection scheme. In: Lemke, H.U., Vannier, M.W., Inamura, K., Farman, A.G., Doi, K. (eds.): Cars 2000. Computer Assisted Radiology and Surgery. Elsevier Science, Amsterdam (2000) 1038.

3. Sahiner, B., Chan, H.P., Petrick, N., Helvie, M.A., Goodsitt, M.M.: Computerized characterization of masses on mammograms: The rubber band straightening transform and texture analysis. Med Phys 25 (1998) 516-526. 